Г.В.Федорович

Роль инерционного механизма в процессе очистки воздуха
в легких от аэрозольных частиц

000 "НТМ-Защита": 115201, Москва, Каширское ш., 22, корп. 4, стр. 7

\title{
G.V.Fedorovich \\ A role of inertial mechanism for clearance of aerosol particle from the lung
}

Key words: lungs, flow turbulence, sedimentation of particles, airway deposition.

Ключевые слова: легкие, завихрение потоков, осаждение частиц, распределение в дыхательных путях.

\begin{abstract}
Аэрозоли в легких - это задача, в результате решения которой возможно избежать многих проблем в самых различных областях. Например, в медицинских приложениях: терапия с использованием аэрозолей позволяет подавать лекарства прямо в те отделы легких, где оно должно действовать. В работе С.Ю.Федорова $u д p$. [1] при решении проблемы оптимальной доставки аэрозольных препаратов в дистальные бронхи авторы опирались на математическую модель системы дыхания. Ингаляционные устройства представляются наиболее логичными и эффективными [2].

Осаждение ингалированных аэрозолей может быть также использовано как инструмент для неинвазивного определения изменений в дыхательных путях легких при различного рода патологиях - эмфиземе, астме, бронхите и др.

Выполнено много исследований (как экспериментальных, так и теоретических) осаждения частиц [3-5]. Основные механизмы осаждения - инерционное столкновение, седиментация (оседание) и диффузия [6]. На основании работ [7-9], в которых обнаружено явление завихрения ламинарных потоков в местах бифуркаций дыхательных путей трахеобронхиального дерева (ТБД), в данной статье основное внимание уделено инерционному механизму очистки воздуха. Вторичные завихренные потоки играют критическую, но не совсем понятную роль в фильтрации определенных частиц из вдыхаемого воздуха.

Рассмотрим подробнее, как работает инерционный механизм в завихрениях воздушного потока в дыхательных путях легких.
\end{abstract}

\section{Характеристики завихренных потоков}

Существуют многочисленные технические приложения (усиление тепло- и массообмена, работа измерительных приборов), в которых вихрь либо желателен, либо представляет собой помеху. Общие результаты, описывающие поведение вихрей в каналах с ламинарным потоком, приведены в работе
T.F.Ayinde [10]. Численно решались уравнения Навье-Стокса для стационарного 3-мерного осесимметричного течения. Вычислялось распределение завихренности вдоль оси канала. Распределение аксиальной скорости на входе можно принять равномерным. Найдено, что вниз по потоку у стенок образуется пограничный слой Прандтля, толщина которого постепенно нарастает и в достаточно длинных каналах на некотором расстоянии от входа пограничный слой заполняет все поперечное сечение. На расстоянии Le (его принято назвать входной длиной) от входа в канал формируется течение Пуайзеля. Для Le справедлива оценка: Le $\approx 0,2 \times \mathrm{R} \times \mathrm{Re}$, где Re - число Рейнольдса, определенное через радиус $\mathrm{R}$ канала и скорость воздуха $\mathrm{V}_{\text {in }}$ на входе в канал: $\mathrm{Re}=\mathrm{R} \times \mathrm{V}_{\text {in }} / v$, где $v-$ кинематическая вязкость.

Для дыхательных путей в легких характерно отношение длины Lc к радиусу, близкое к 6 [7]. Можно утверждать, что в верхних дыхательных путях, где $\operatorname{Re}>30$, течение Пуайзеля сформироваться не успевает.

Для случая небольшой (по сравнению с радиусом $\mathrm{R}$ канала) толщины пограничного слоя аксиальная скорость $V_{z}$ в потоке описывается соотношением (решение Блазиуса):

$$
\mathrm{V}_{\mathrm{z}}=\mathrm{V}_{\text {in }} \times \mathrm{f}(\zeta)
$$

где $V_{\text {in }}$ - скорость вдали от стенки, ее можно приравнять к скорости на входе в канал; $\zeta$ - автомодельная переменная.

$$
\zeta=(\mathrm{R}-\mathrm{r}) \times\left[\mathrm{V}_{\text {in }} / \mathrm{z} \times \mathrm{v}\right]^{1 / 2} .
$$

Функция $\mathrm{f}(\xi)$ представляет собой результат численного решения уравнения, следующего из уравнений непрерывности и Навье-Стокса при переходе к автомодельной переменной $\zeta$. Для практических расчетов можно описывать ее поведение простой зависимостью $(\xi \circ \approx 3)$ :

$$
\mathrm{f}(\zeta) \approx \zeta /(\zeta+\zeta \mathrm{o})
$$


сохраняющей особенности поведения $\mathrm{f}(\xi)$ и одновременно позволяющей проинтегрировать уравнение динамики элементарных объемов газа. Например, использование соотношений (1) и (3) дает возможность определить закон движения элементарного объема вдоль канала. Для этого целесообразно ввести безразмерные переменные:

$$
\mathrm{x}=\mathrm{z} / \mathrm{R} ; \mathrm{y}=\mathrm{r} / \mathrm{R} ; \tau=\mathrm{t} \times \mathrm{V}_{\text {in }} / \mathrm{R} \text {. }
$$

Через эти переменные автомодельная переменная $\zeta$ будет выражена формулой:

$$
\zeta=(1-\mathrm{y}) \times[\operatorname{Re} / \mathrm{x}]^{1 / 2} .
$$

Уравнение движения частиц вдоль продольной оси канала описывается уравнением:

$$
\mathrm{dx} / \mathrm{d} \tau=\mathrm{f}(\zeta) \approx \zeta /(\zeta+\zeta \mathrm{o})
$$

Предполагая, что расстояние у $=\mathrm{y}_{1}$ до оси канала остается неизменным при движении воздуха, получим:

$$
\tau=\mathrm{x} \times\left[1+(2 / 3) \zeta \mathrm{o} \times(\mathrm{x} / \mathrm{Re})^{1 / 2} /\left(1-\mathrm{y}_{1}\right)\right] .
$$

Это решение допускает простую интерпретацию. Если $v \rightarrow 0$, то $\operatorname{Re} \rightarrow \infty$ и 2-й член в квадратных скобках мал. При этом $\tau \approx$ х, т. е. независимо от начального радиуса входа элемента объема воздуха в канал, он выходит из канала (при х $=\mathrm{x}_{\mathrm{c}} \equiv \mathrm{Lc} / \mathrm{R}$ ) через время (безразмерное) $\tau_{\mathrm{c}} \approx \mathrm{X}_{\mathrm{c}}$. Скорость (безразмерная) движения по каналу равна 1 . Наличие пристеночного пограничного слоя сказывается на увеличении времени прохода. При приближении к стенке $\zeta$ можно считать малой (по сравнению с $\zeta$ ), здесь для расчета траекторий можно пользоваться приближенным уравнением:

$$
\mathrm{dx} / \mathrm{d} \tau \approx \zeta / \zeta \mathrm{o},
$$

решение которого имеет вид:

$$
\tau=(2 / 3) \zeta_{0} \times \mathrm{x}^{3 / 2} /\left[\operatorname{Re}^{1 / 2}\left(1-\mathrm{y}_{1}\right)\right],
$$

т. е. поправка к решению $\tau \approx \mathrm{x}$ для центральной области канала становится основной для близких к стенке траекторий.

Распределение азимутальной скорости $\mathrm{V} \varphi$ по радиусу в сечении канала хорошо интерполируется функцией:

$$
\mathrm{V}_{\varphi}=\Gamma \times \mathrm{V}_{\text {in }} \times(\mathrm{r} / \mathrm{R}) \times(1-\mathrm{r} / \mathrm{R}) .
$$

Коэффициент Г, определяющий амплитуду $\mathrm{V} \varphi$, убывает с расстоянием z вдоль потока по экспоненциальному закону с характерной длиной Le. Для затухания вихря справедливы те же утверждения, что и для формирования течения Пуайзеля: в верхних дыхательных путях затухание мало.

\section{Траектории аэрозольных частиц в завихренных потоках в цилиндрических каналах}

Движение аэрозольных частиц происходит под действием вязких сил. Характер движения определяется числом Стокса (Stk):

$$
\text { Stk }=(1 / 18) \times\left(\rho_{p} / \rho_{\mathrm{a}}\right) \times\left(\mathrm{d}_{\mathrm{p}}^{2} / \mathrm{v}\right) \times\left(\mathrm{V}_{\text {in }} / \mathrm{R}\right) .
$$

Здесь $\rho_{\mathrm{p}}$ и $\rho_{\mathrm{a}}-$ плотность частиц и воздуха соответственно, $d_{p}$ - диаметр частиц. В дыхательных путях легких число Стокса для частиц микронного размера обычно невелико $(<0,1)$, что свидетельствует о вовлеченности их в движение воздуха. Например, аксиальная и азимутальная скорости воздуха и частиц одинаковы, радиальная скорость $\mathrm{U}_{\mathrm{r}}$ частиц с массой т определяется уравнением:

$$
\mathrm{m} \times \mathrm{V}_{\varphi}^{2} / \mathrm{r}=3 \times \pi \times \mathrm{d}_{\mathrm{p}} \times \rho_{\mathrm{a}} \times \mathrm{v} \times \mathrm{U}_{\mathrm{r}} .
$$

Использование для $\mathrm{V} \varphi$ выражения (10) и введение согласно (4) безразмерной переменной у приводит к решению для $\mathrm{U}_{\mathrm{r}}$ вида:

$$
\mathrm{U}_{\mathrm{r}}=\mathrm{V}_{\text {in }} \times \Gamma^{2} \times \text { Stk } \times \mathrm{y} \times(1-\mathrm{y})^{2} .
$$

Таким образом, решение задачи о распределении скоростей в закрученном потоке непосредственно определяет скорости аэрозольных частиц в этом потоке.

Эффект выноса аэрозолей на периферию потока приводит к эффективному механизму очистки воздуха за счет захвата частиц в бронхиолах ТБД. В пристеночном слое частицы двигаются медленнее основного потока, и когда этот эффект становится значительным, за время вдоха или выдоха частицы не успевают пройти ту ветвь ТБД, где они замедлились. В следующем цикле дыхания частицы будут вынесены еще ближе к стенке, их продольная скорость станет еще меньше и частицы останутся в захватившей их ветви сколь угодно долго, пока не произойдет их осаждение за счет механизма, возможно недостаточно эффективного для осаждения за 1 проход.

Опишем эффект захвата частиц количественно.

\section{Время пролета канала. Эффект захвата частиц в легких}

Уже была проводена оценка времени прохода канала элементарным объемом воздуха и / или захваченной в поток частицей по траектории, проходящей на постоянном расстоянии (безразмерном) у от центра канала. Учтем эффект выноса частиц к стенкам канала. Интересно движение в пограничном слое, поэтому для функции х $(\tau)$ будет использоваться уравнение (8). Для у $(\tau)$ уравнение следует из (13):

$$
\mathrm{dy} / \mathrm{d} \tau=\Gamma^{2} \times \text { Stk } \times \mathrm{y} \times(1-\mathrm{y})^{2} .
$$

Система уравнений (8) и (14) определяет (r, z)траектории [в безразмерных переменных (х, у)-траектории]. Полная траектория получится после закручивания (r, z)-траекторий вокруг оси канала в соответствии с вращением частиц с азимутальной скоростью $\vee \varphi$ вместе с потоком.

Решение у (х) имеет следующий вид:

$$
\begin{gathered}
\mathrm{y}(\mathrm{x})=\mathrm{y}_{1} /\left\{\mathrm{y}_{1}+\left(1-\mathrm{y}_{1}\right) \times \exp \left[-(2 / 3) \times \Gamma^{2} \times\right.\right. \\
\left.\left.\mathrm{Stk} \times \zeta \mathrm{O} \times \mathrm{x}^{3 / 2} / \operatorname{Re}^{1 / 2}\right]\right\} .
\end{gathered}
$$


Оценку времени пролета можно получить, проинтегрировав (14):

$$
\begin{gathered}
\Gamma^{2} \times \operatorname{Stk} \times \tau=\ln \left[\mathrm{y} \times\left(1-\mathrm{y}_{1}\right) / \mathrm{y}_{1} \times(1-\mathrm{y})\right]+ \\
1 /(1-\mathrm{y})-1 /\left(1-\mathrm{y}_{1}\right) .
\end{gathered}
$$

Если в (16) подставить $\mathrm{yc}_{\mathrm{c}}=\mathrm{y}\left(\mathrm{x}=\mathrm{x}_{\mathrm{c}}\right)$, то получим оценку времени пролета канала с учетом выноса частицы в зону медленного пристеночного течения:

$$
\tau_{\mathrm{c}}=\left\{\lambda+[\exp (\lambda)-1] \times \mathrm{y}_{1} /\left(1-\mathrm{y}_{1}\right)\right\} / \Gamma^{2} \times \text { Stk },
$$

где: $\lambda=(2 / 3) \times \Gamma^{2} \times \mathrm{Stk} \times \xi$ o $\times \mathrm{X}_{\mathrm{c}}^{3 / 2} / \operatorname{Re}^{1 / 2}$. При $\lambda>1$ время пролета растет экспоненциально и может достичь значений, больших, чем время (безразмерное) вдоха. Это можно считать эффектом захвата частицы в соответствующем канале. Как видно из (17), величина

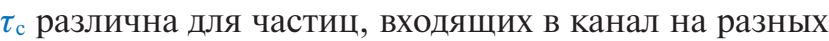
расстояниях $\mathrm{y}_{1}$ от центра канала. Захватываются только те частицы, которые входят в канал на расстояниях больших, чем $\mathrm{y}_{1}$, определяемое из уравнения:

$$
\mathrm{y}_{1} /\left(1-\mathrm{y}_{1}\right)=\left[\Gamma^{2} \times \text { Stk } \times \tau_{\text {вд }}-\lambda\right] /[\exp (\lambda)-1] .
$$

Соответственно, вероятность захвата будет определяться формулой:

$$
P=\frac{2 \pi \int_{y_{1}}^{1} y d y}{2 \pi \int_{0}^{1} y d y}=1-y_{1}^{2} .
$$

Знаменатель I (0) в этой формуле введен для нормировки вероятности на единицу. Соотношения (18) и (19) дают возможность вычислить вероятность захвата аэрозольных частиц в дыхательных путях различных генераций ТБД.

\section{Иллюстрация работы механизма на модели ТБД}

Для приложения полученных результатов к процессам в легких необходимо в соотношения (14-16) подставить значения чисел Рейнольдса и Стокса, характерные для потоков в дыхательных путях.

Здесь необходимо использовать подходящую модель реального ТБД. В настоящее время разработано и используется множество моделей ТБД [7], они различаются в зависимости от целей работы. Для наших целей подходит простая морфометрическая модель детерминированного симметричного дихотомического ветвления дыхательных путей с пропорциональным уменьшением их размеров, т. е. с сохранением подобия ветвей [11]. Номер і-генерации в этой модели указывает на параметры воздушного канала, а не на его место в иерархической структуре. Однако параметры модели по возможности выбраны так, что с ее помощью можно указать на реальную локализацию тех или иных процессов.

Количество N (i) ветвей і-генерации, диаметр d (i) и скорости воздуха v (i) в них задаются формулами:

$$
\mathrm{N}(\mathrm{i})=2^{\mathrm{i}} ; \mathrm{d}(\mathrm{i})=\mathrm{d}(0) \times 2^{-\mathrm{i} / 3} ; \mathrm{v}(\mathrm{i})=\mathrm{v}(0) \times 2^{-\mathrm{i} / 3} \text {. }
$$

Нулевая ветвь - трахея $[\mathrm{d}(0)=1,44 \mathrm{~cm} ; \mathrm{v}(0)=290$ см / c], уровню $\mathrm{i}=18$ соответствуют терминальные бронхиолы. Длина I-каналов предполагается втрое большей, чем диаметр для всех генераций. В табл. 1 приведены числа Рейнольдса, характеризующие потоки воздуха в дыхательных путях.

Таблица 1

Числа Рейнольдса в ветвях ТБД

$\begin{array}{lccccccccccc}i & 0 & 2 & 4 & 6 & 8 & 10 & 12 & 14 & 16 & 18 & 20 \\ \text { Re } & 1392,0 & 552,4 & 219,2 & 87,0 & 34,5 & 13,7 & 5,4 & 2,2 & 0,9 & 0,3 & 0,1\end{array}$

Видно, что практически во всех ветвях ТБД течение ламинарное, однако c $\operatorname{Re}>1$. В принятой модели время $\mathrm{t}_{\mathrm{c}}$ прохождения воздушного канала одинаково для всех генераций:

$$
\mathrm{t}_{\mathrm{c}}=1 \text { (i) } / \mathrm{v}(\mathrm{i})=3 \times \mathrm{d}(\mathrm{i}) / \mathrm{v}(\mathrm{i})=3 \times \mathrm{d}(0) / \mathrm{v}(0) \text {. }
$$

Подставляя сюда значения d $(0)$ и v (0), получим время: $\mathrm{t}_{\mathrm{c}}=0,005 \mathrm{c}$. Можно сопоставить это время с характерным временем вдоха $\mathrm{t}_{\text {вд }}=1,25$ с (считаем, что периодичность дыхания 12 мин $^{-1}$ и на каждый период приходится 4 одинаковой длительности фазы: вдох, пауза, выдох, пауза). Отношение этих величин $t_{\text {вд }} / t_{c}=250$ можно рассматривать как возможное количество генераций ТБД, которое воздух мог бы пройти за время вдоха. Как и следовало ожидать, это количество значительно больше полного числа генераций $(\approx 25)$ ТБД, т. е. прохождение дыхательных путей до уровня альвеол занимает не более $10 \%$ всего времени вдоха.

В принятой модели ТБД отношение скорости воздуха к диаметру одинаково для всех генераций, поэтому число Стокса одинаково во всех ветвях, оно меняется в зависимости от диаметра частиц. Соответствующие результаты приведены в табл. 2.

Таблица 2

Числа Стокса для частищ, различных диаметров

$\begin{array}{lccccccc}d_{p}, \text { MKM } & 1 & 2 & 4 & 6 & 10 & 20 & 40 \\ \text { Stk } & 0,0015 & 0,0060 & 0,024 & 0,054 & 0,149 & 0,60 & 2,39\end{array}$

В соответствии с полученными результатами частицы с диаметром до 20 мкм (Stk $<1)$ вовлекаются в движение воздуха. Только частицы с большим диаметром могут "оторваться" от потока и, например, осесть на стенку непосредственно за счет инерционного механизма.

Перенос результатов по захвату аэрозолей на дыхательные пути ТБД осуществляется подстановкой в выражения чисел Рейнольдса из табл. 1, соответствующих і-генерации ТБД. При этом параметр $\lambda$ становится функцией номера і-генерации. В соответствии с (18) функцией номера становится и радиус входа захватываемых частиц, и вероятность захвата Р, вычисляемая по (19). Диаметр частицы определяет число Стокса.

Результаты расчета вероятности захвата частиц различных диаметров в генерациях дыхательных путей ТБД представлены на рис. 1.

Видно, что механизм очистки воздуха за счет захвата аэрозолей в дыхательных путях достаточно эф- 


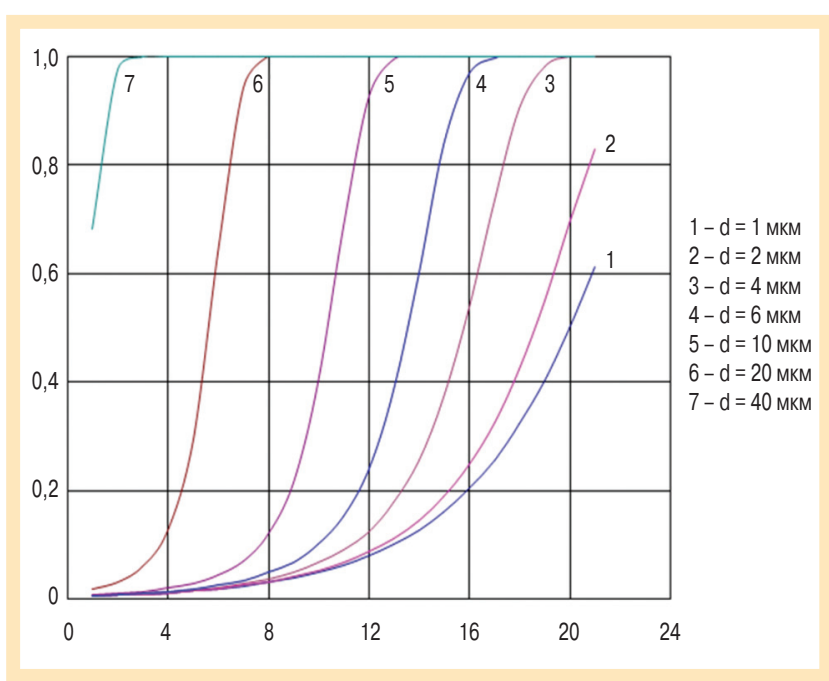

Рис. 1. Зависимость вероятности захвата частицы диаметром d в дыхательных путях і-генерации ТБД

фективен даже для частиц с диаметром 1-2 мкм. Частицы диаметром $\geq 4$ мкм практически достоверно захватываются в каналах с номером генерации $<19$, т. е. до респираторного отдела. Частицы больших диаметров (> 10 мкм) захватываются в верхних дыхательных путях - трахее, зональных внелегочных бронхах, внутрилегочных субсегментарных бронхах.

Рассчитанная по формуле (19) вероятность захвата определяет долю захваченных частиц от числа частиц, вошедших в рассматриваемый канал. Можно определить вероятность Ра (i) достижения дыхательных путей і-генерации как долю частиц, попавших в них от числа частиц, вошедших в нулевую генерацию (трахею) ТБД. Очевидно:

$$
\mathrm{P}_{\mathrm{a}}(i)=\prod_{k=0}^{k=i-1}[1-\mathrm{P}(k)] .
$$

Здесь произведение вычисляется для всех сомножителей от $\mathrm{k}=0$ до $\mathrm{k}=\mathrm{i}^{-1}$. Функция $\mathrm{P}_{\mathrm{a}}$ (i) для аэрозольных частиц разных диаметров приведена на рис. 2.

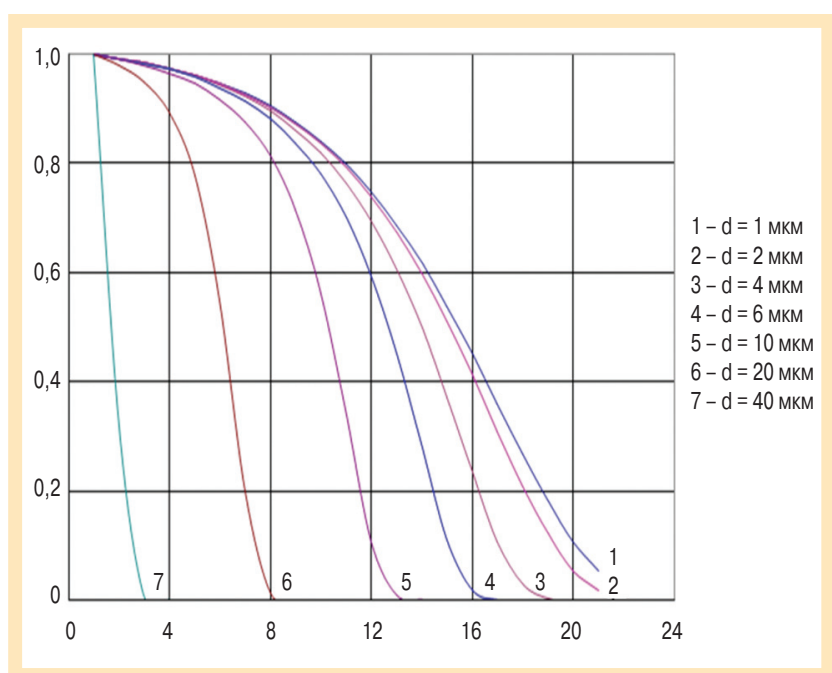

Рис. 2. Вероятность для аэрозольных частиц достичь дыхательных путей і-генерации ТБД

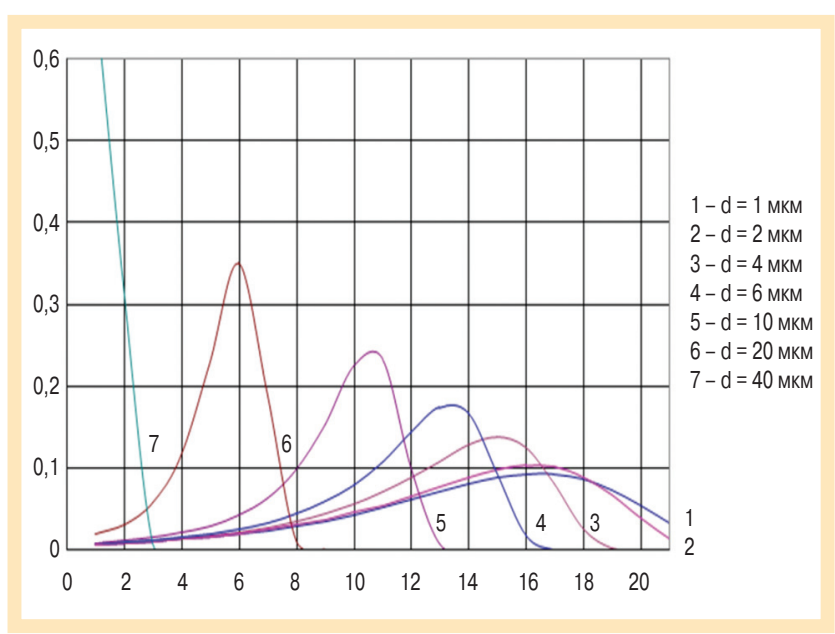

Рис. 3. Распределение осевших частиц по номерам генерации ТБД

Видно, что вероятность достичь глубоких отделов легких практически равна нулю для частиц размерами $>10$ мкм. Частицы с размерами 4-6 мкм могут проникнуть в легкие только до терминальных бронхиол. Только частицы размером $<2$ мкм могут проникать в респираторные отделы легких, но коэффициент очистки для них составляет порядок и более. Эти данные также свидетельствуют о высокой эффективности захвата частиц как механизма очистки воздуха от аэрозолей.

Приведем данные о вероятности захвата частиц в дыхательных путях ТБД, отнесенной к частицам, вошедшим в трахею. Обозначая эту величину как $\mathrm{P}_{\mathrm{c}}(\mathrm{i})$, можем рассчитать ее по очевидной формуле:

$$
\mathrm{P}_{\mathrm{c}}(\mathrm{i})=\mathrm{Pa}_{\mathrm{a}}(\mathrm{i}) \times \mathrm{P}(\mathrm{i}) \text {. }
$$

Результаты расчета величины $\mathrm{P}_{\text {c }}$ (i) приведены на рис. 3.

Последний результат дает наглядное представление о том, в каких областях легких происходит захват аэрозольных частиц различных диаметров. Видно, что даже самые мелкие частицы в основном захватываются в бронхиолах до респираторного отдела. Крупные частицы (диаметром $\geq 6$ мкм) вообще не проникают до терминальных бронхиол.

\section{Заключение}

Появившиеся недавно работы, в которых обнаружено явление завихрения ламинарных потоков в местах бифуркаций дыхательных путей ТБД, послужили основанием для уточнения инерционный механизм очистки воздуха в легких от аэрозольных частиц. Для описания вихрей в каналах с ламинарным потоком, использовались известные результаты, полученные методами вычислительной газодинамики.

В работе рассчитаны вероятность захвата за счет инерционного механизма в отдельном воздушном канале и вероятность захвата, отнесенная ко всем частицам, попавшим в трахею, и далее - в дыхательные пути ТБД.

Обнаружено, что механизм очистки воздуха за счет захвата аэрозолей в дыхательных путях достаточно 
эффективен даже для частиц диаметром 1-2 мкм. Частицы диаметром $\geq 4$ мкм практически достоверно захватываются в каналах с номером генерации $<19$, т. е. до респираторного отдела. Частицы больших диаметров (> 10 мкм) захватываются в верхних дыхательных путях - трахее, зональных внелегочных бронхах, внутрилегочных субсегментарных бронхах. Только частицы с размером $<2$ мкм могут проникать в респираторные отделы легких, но коэффициент очистки для них составляет порядок и более. Эти данные свидетельствуют о высокой эффективности захвата частиц как механизма очистки воздуха от аэрозолей.

\section{Литература}

1. Федоров С.Ю., Никаноров Б.А., Хадариев А.А. и др. К математическому и физическому моделированию процесса ингаляции в системе ингалятор-респираторный тракт. Пульмонология 1995; 3: 20-27.

2. Авдеев С.Н. Устройство доставки ингаляционных препаратов, используемые при терапии заболеваний дыхательных путей. Рус. мед. журн. Пульмонология. Аллергология 2002; 10 (5): 255-261.

3. Hofmann W., Mainelis G., Mohamed A. et al. Modeling approaches in current lung dosimetry models. Environ. Int. 1996; 22 (Suppl. 1): S965-S976.
4. Musante C.J., Martonen T.B. Computer simulations of particle deposition in the developing human lung. J. Air Waste Manag Assoc. 2000; 50: 1426-1432.

5. James A.C. Lung dosimetry. In: Nazaroff W.W., Nero A.V., eds. Radon and its decay products in indoor air. New York: Wiley-Interscience; 1988. 259-309.

6. Swift D.L. Aerosol characteristics and generation. In: Moren F., Dolovich M.B., Newhouse M.T. et al., eds. Aerosols in medicine: Principles, diagnosis and therapy. New York: Elsevier Science (Biomedical Division); 1985. 53-76.

7. Gemci T., Ponyavin V., Chen Y. et al. Computational model of airflow in upper 17 generations of human respiratory tract. J. Biomech. 2008; 41: 2047-2054.

8. Kabilan S., Lin C.-L., Hoffman E.A. Characteristics of airflow in a CT-based ovine lung: A numerical study. J. Appl. Physiol. 2007; 102: 1469-1482.

9. Lee J.W., Goo J.H., Chung M.K. Characteristics of inertial deposition in a double bifurcation. J. Aerosol Sci. 1996; 27: 119-138.

10. Ayinde T.F. A generalized relationship for swirl decay in laminar pipe flow. Sadhana 2010; 35 (2): 129-137.

11. Yeh H.C., Schum G.M. Models of human lung airways and their applications to inhaled particle deposition. Bull. Math. Biol. 1980; 42: 461-480.

\section{Информация об авторе}

Федорович Геннадий Викторович - д. Ф.-М. Н., технический директор ООО "НТМ-Защита"; тел.: (495) 500-03-00; факс: (495) 500-03-00; e-mail: fedorgv@gmail.com 